\title{
Influence of forced convection on the evaporation and internal dynamics inside of an array of salt solution droplets
}

\author{
Omar Al-Rawi ${ }^{1,2, *}$, and Mark Wilson ${ }^{1}$ \\ ${ }^{1}$ School of Mechanical Engineering, University of Leeds, Leeds, LS2 9JT, UK \\ ${ }^{2}$ State Company for Oil Projects, Ministry of Oil, Baghdad, Iraq
}

\begin{abstract}
The effects of a gentle forced air convection on the internal dynamics of an array of multiple pinned sessile salt solution droplets are investigated via fully-coupled transient ALE finite element analysis. Results highlight the competition between the shear-induced circulation within the droplets and the gravity-driven flow in the droplets arising from increasing liquid density in regions of high water evaporation. At low air speeds, gravity effects dominate, resulting in a non-uniform concentration distribution. However, at higher speeds the shear-induced circulation within the droplets becomes sufficient to mix the liquid within the droplets via a 3D flow pattern, resulting in greater concentration uniformity. In addition, the shielding effect of leading droplets on downstream droplets is explored for various air speeds, with results showing differences in average concentration levels.
\end{abstract}

\section{Nomenclature}

$\begin{array}{lll}\bar{c}, \bar{C} & \mathrm{~mol} / \mathrm{m}^{3} & \text { solute, vapour concentration } \\ \mathbf{j}_{\Gamma} & \mathrm{mol} / \mathrm{m}^{2} \mathrm{~s} & \text { total molar flux } \\ \rho_{l,}, \rho_{v} & \mathrm{~kg} / \mathrm{m}^{3} & \text { liquid, vapour water density } \\ \mathbf{n} & & \text { unit surface normal vector } \\ \mathbf{T} & \mathrm{Pa} & \text { stress tensor } \\ D_{s}, D_{v} & \mathrm{~m}^{2} / \mathrm{s} & \text { solute, vapour diffusivity }\end{array}$

\section{Introduction}

In recent decades, the dynamics inside multiple droplets evaporating close to each other has attracting interest in many industrial and scientific applications such as surface coatings [1], inkjet printing [2], and pharmaceuticals [3]. Experimental and numerical studies show that the dynamics inside a droplet having a pinned contact line depend on the evaporation conditions and the environment surrounding the droplet [4]. An outward flow towards the edge of the droplet producing a ringlike deposit is observed, which is caused by either the replenishment of liquid lost in this area or by edge enhanced evaporation $[4,5]$. In contrast, a flow towards the centre of the droplet inhibiting the ring-like deposit is generated due to either centre enhanced evaporation or surface tension gradients causing Marangoni flow [6].

In droplets of evaporating aqueous solutions, another flow mechanism is observed. Kang et al. [7], Savino and Monti [8], Lee et al. [9], and Pradhan and Panigrahi [10, 11] found that due to the concentration gradients inside the droplet, buoyancy-driven convection is induced where the liquid moves along the droplet surface towards the contact line and towards the droplet apex in the central region of the droplet. Further observations reveal that this internal flow field is significantly affected by the solute concentration inside evaporating droplets [12].

The interaction between adjacent evaporating droplets via their shared vapour space affects the evaporation behaviour, and hence the flow patterns inside these droplets [13]. Davoust and Theisen characterised experimentally the evaporation rate of a population of droplets in a microsystem [14, 15]. Recently, Pradhan and Panigrahi [10] observed how the fluid convection inside an evaporating droplet is strongly affected by the influence of an adjacent droplet and the distance between them.

The importance of vapour/air convection, i.e. natural and forced, in the evaporation of sessile droplets has also been explored. Experimental work by Kelly-Zion et al. [16] examined the crucial role of natural convection in increasing the evaporation rates of various liquids (3methylpentane, hexane, cyclohexane, and heptane) on an aluminium substrate. Saada et al. [17] observed numerically the effect of buoyant vapour convection on evaporation rates for different droplet radii. The experimental findings for salt solution droplet evaporation show the importance of gas convection around these droplets, which is significantly influenced by the salt concentration, and neglecting this mechanism leads to underestimation of evaporation rates $[18,19]$.

Numerically, Raghuram et al. [20] found that the flow-field inside the droplet induced by Marangoni effects is greatly affected by the forced convective environment. Doursat et al. [21] studied the evolution of the mass and heat transfer during the evaporation of multiple pure water droplets under forced convective environment. Chakraborty et al. [22] analysed the feasibility of using an array of evaporating droplets to dissipate the heat released from microprocessors. They noticed that providing a fan to generate forced air flow in order to remove the diffusive vapour between the

* Corresponding author: o.alrawi@ hotmail.com 
droplets would improve the evaporation rates of these droplets.

Though the above studies illustrate the importance of considering convection in the evaporation of droplets in pure water or binary solvent droplets, it appears that there are no prior studies of the effects of forced convection on the internal dynamics during evaporation of neighbouring salt solution droplets. This is therefore the focus of the present work.

\section{Mathematical model}

The system considered is a semi-infinite regular array of sessile salt solution droplets, over which a laminar airflow passes, as shown in Figure 1. The repeating pattern of the geometry is exploited to reduce the computational domain to a unit flow consisting of two droplets aligned parallel with the airflow, with periodic conditions along the side boundaries.

\subsection{Assumptions}

Droplets of aqueous $\mathrm{NaCl}$ solution with initial volume 2.1 $\mu \mathrm{l}$ and $1 \mathrm{M}$ concentration are used to investigate the effect of the forced convective environment on the solute distribution inside the droplets. For this type of salt solution, the experimental observations of Kang et al. [7] show that, in the absence of an overlying airflow, the flow inside the droplet is driven by convection due to density gradients as a results of increasing salinity, and Marangoni effects are negligible. The system is also assumed to be in thermal equilibrium and thermal effects are neglected. Hence surface tension gradients are ignored here. The effect of gravity is captured via the Boussinesq approximation.

The contacts lines of the droplets are assumed to be pinned, with an initial contact angle of $90^{\circ}$, but the free surfaces otherwise evolve in a fully transient manner in response to local mass loss due to water evaporation. Both liquid and air are modelled as incompressible.

\subsection{Governing equations}

Based on the above assumptions, the species transport, continuity and momentum equations are:

$$
\begin{aligned}
\frac{\partial c}{\partial t}+\boldsymbol{u} \cdot \nabla \bar{c} & =\nabla \cdot(D \nabla \bar{c}) \\
\nabla \cdot \mathbf{u} & =0
\end{aligned}
$$

$\left.\rho\left(\frac{\partial \mathbf{u}}{\partial t}+(\mathbf{u} \cdot \nabla) \mathbf{u}\right)=\nabla \cdot\left[-p \mathbf{I}+\mu\left(\nabla \mathbf{u}+(\nabla \mathbf{u})^{\mathbf{T}}\right)\right]-\Delta \rho g \mathbf{B}\right)$ where $\bar{c}$ is the molar concentration field and $D$ is the

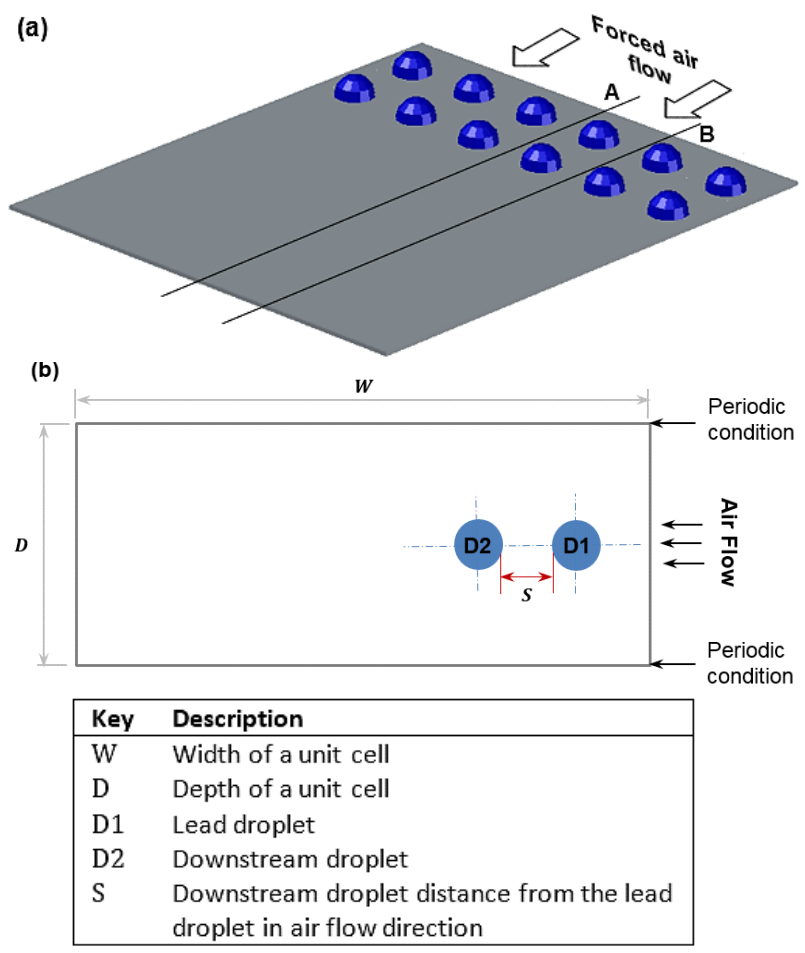

Fig. 1. Sessile droplet array considered (a), and plan view of the representative computational domain (b).

diffusion coefficient in the relevant fluid.

\subsection{Initial and boundary conditions}

The droplets are initially hemispherical, with a uniform solute concentration of $1 \mathrm{M}$. During evaporation, the solute concentration $\bar{c}$ increases at the droplet free surfaces, and diffuses to the inner regions of the droplets. At the free surfaces, the condition on the solute concentration is therefore

$$
-D_{s} \nabla \bar{c} \cdot \mathbf{n}=-\frac{\bar{c} \mathbf{j}_{\Gamma}}{\rho_{l}} \bar{M}_{H_{2} O}
$$

where

$$
\mathbf{j}_{\Gamma}=-D_{v} \nabla \bar{C}+\mathbf{u} \bar{C}
$$


is local total molar flux of water vapour at the free surface and $\bar{C}$ is the molar water vapour concentration. At the droplet surfaces, the concentration of water vapour is assumed to be saturated, with an adjustment for the solute presence via Raoult's law [10], hence $\bar{C}=0.93$ $\mathrm{mol} / \mathrm{m}^{3}$ at the free surfaces. At the outer boundaries, the vapour concentration is also fixed in terms of saturation conditions, but assuming a relative humidity of $60 \%$ and ambient temperature of $20^{\circ} \mathrm{C}$. The velocity and stress conditions at the free surfaces are given by:

$$
\begin{gathered}
\mathbf{u}_{v}-\mathbf{u}_{\imath}=\bar{M}_{H_{2}} o\left(1 / \rho_{n}-1 / \rho_{l}\right)\left(\mathbf{j}_{\Gamma} \cdot \mathbf{n}\right) \mathbf{n} \\
\mathbf{n} \cdot \mathbf{T}_{v}-\mathbf{n} \cdot \mathbf{T}_{\imath}=\sigma(\nabla \cdot \mathbf{n}) \mathbf{n}
\end{gathered}
$$

where $\mathbf{T}$ is the stress tensor, and the subscripts $v$ and $l$ on $\mathbf{u}$ and $\mathbf{T}$ refer to the values of these entities on the vapour and liquid sides of the interface respectively. Condition (6) accounts for the increase in specific volume of water as it evaporates, resulting in a Stefan flow, while condition (7) incorporates both the capillary pressure and shear stress of the airflow acting on the liquid.

At fluid-solid boundaries, the no-slip and no-flux conditions are applied, while the airflow inlet is a fullydeveloped laminar flow with entrance length $0.06 R e D_{H}$. At the outlet, $-\mathbf{n} \cdot D_{v} \nabla C=0$ and atmospheric pressure constraints are applied. As noted above, periodic conditions are applied along the sides of the domain, which is chosen to have a streamwise length of $60 \mathrm{~mm}$, a spanwise width of $20 \mathrm{~mm}$ and a height of $10 \mathrm{~mm}$.

\subsection{Numerical method}

Equations (1)-(3) were solved subject to the above conditions via a 3D transient Arbitrary LagrangianEulerian finite element approach implemented in COMSOL Multiphysics ${ }^{\circledR}$, v. 5.3. An unstructured quadratic tetrahedral mesh was used, where nodes on the free surfaces were displaced according to the local evaporative mass loss, with a velocity given by

$$
\dot{\mathbf{x}}_{\Gamma}=\left(\mathbf{u}_{\imath} \cdot \mathbf{n}\right) \mathbf{n}-\frac{\left(\mathbf{j}_{\Gamma} \cdot \mathbf{n}\right) \Omega_{H_{2} O}}{\rho_{l}} \mathbf{n} .
$$

Nodes in the interior of the liquid and vapour regions were moved via the Winslow mesh deformation method, subject to equation (8) at the free surface and zero mesh displacement at the outer boundaries.

\section{Results and discussion}

The forced convection air speeds considered here are $U=$ $0.08,0.2$ and $2.0 \mathrm{~m} / \mathrm{s}$. The value $0.08 \mathrm{~m} / \mathrm{s}$ was chosen as it was the lowest non-zero speed for which meshindependent results could be obtained with the available computational resources. Below this value, the velocities on the free surfaces are very small, and this is an area of parameter space that is currently being further explored. The speed was limited to $2 \mathrm{~m} / \mathrm{s}$ to ensure that the air flow remained laminar, and that no displacement of the droplets should be expected [23]. Note that, $3 \mathrm{~mm}$ is considered here as a separation distance between the two droplets.

\subsection{Validation against experiments}

Before using the model to explore the effect of forced convection on the salt solution droplets, its predictions for natural convection (i.e. no imposed airflow) were first tested against the experimental results of Pradhan and Panigrahi [10], with all parameters specified as in Ref. [10]. Figure 2 shows predictions of the velocity

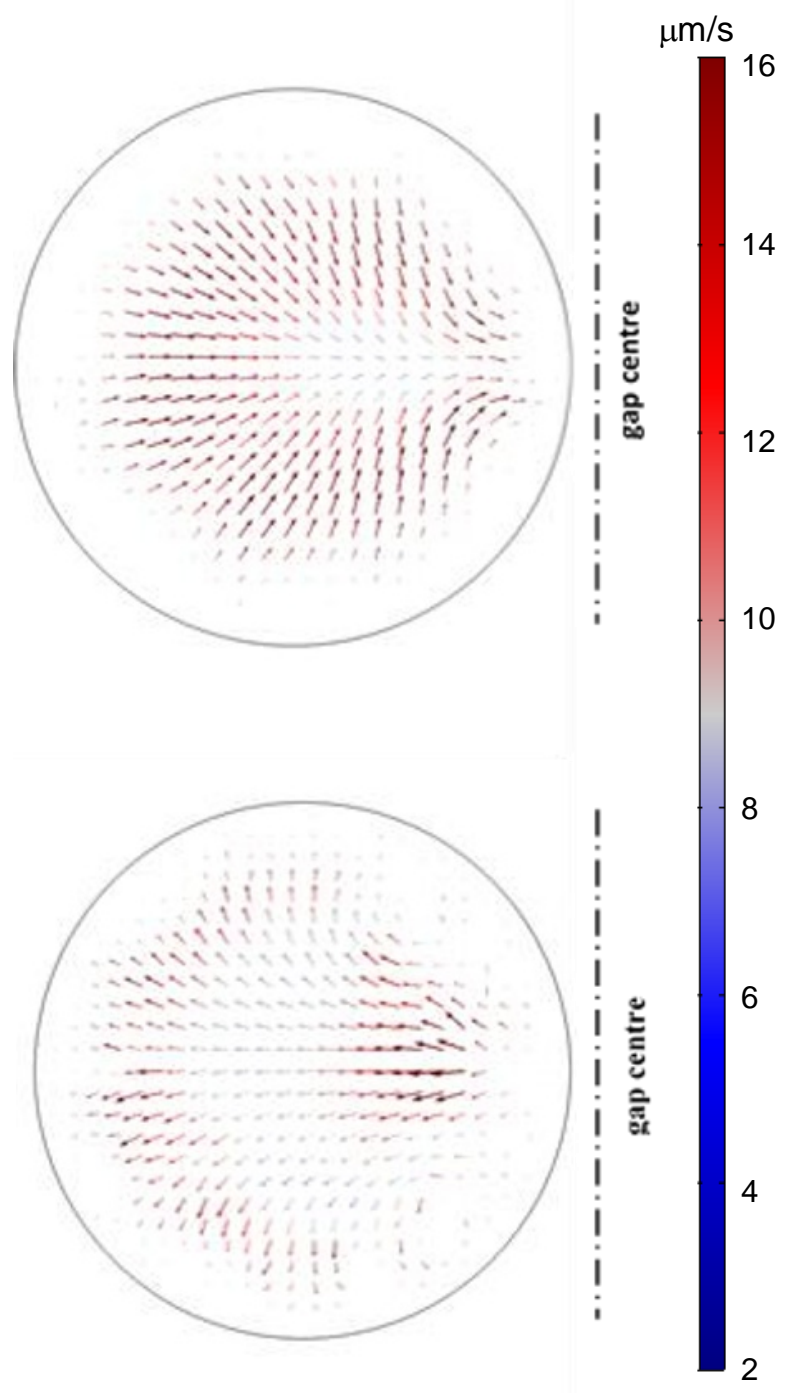

Fig. 2. Numerical results for the velocity field on horizontal places inside the left-hand droplet of two adjacent droplets. The planes shown are at heights (a) $50 \mu \mathrm{m}$ and (b) $350 \mu \mathrm{m}$ above the substrate.

vectors in horizontal planes at heights of 50 and $350 \mu \mathrm{m}$ above the substrate. These show the same general flow patterns as the corresponding experimental velocity fields.

\subsection{Distribution of solute inside droplets}

In a natural convection environment, the salt concentration increases along the droplet free surfaces due to the water evaporation from those surfaces. This induces a gravity-driven flow due to the increase in density with 
increasing salinity, and a non-uniform distribution of solute concentration within the droplets.

The presence of a gentle forced air convection changes the flow and salt distribution inside droplets, as a result of the shear-induced flow within the droplet caused by the passing air. Figure 3 shows the resulting velocity field in the lead (i.e. upstream) droplet on a central vertical streamwise plane, along with the velocity field on the interior side of the free surface (recall that the velocity field has different values on the interior and exterior sides of the free surface, as expressed in

$$
\text { (a) }
$$

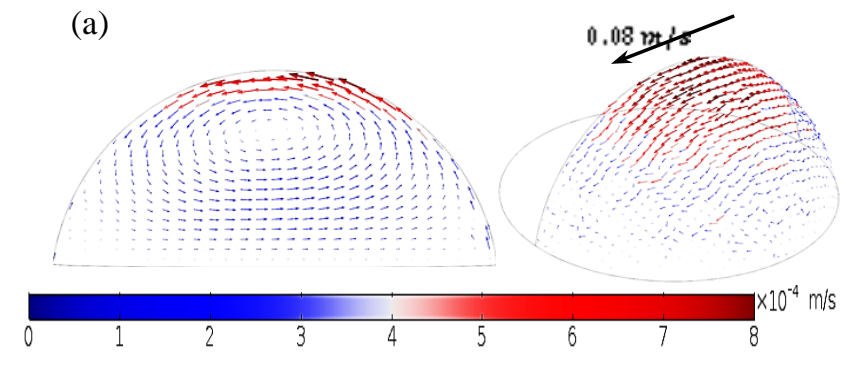

(b)

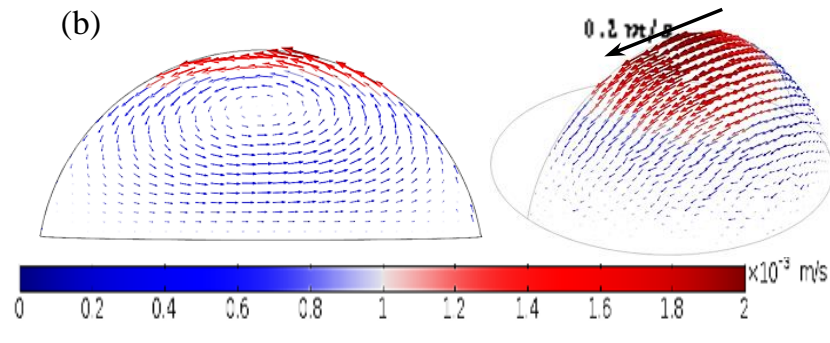

(c)

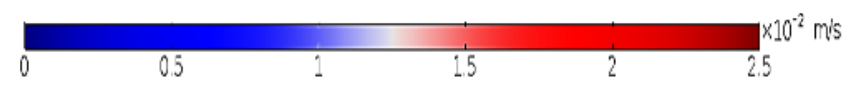

Fig. 3. Left: Velocity field in a streamwise vertical section through the centre of the lead droplet exposed to forced convection at (a) 0.08 , (b) 0.2 , (c) $2 \mathrm{~m} / \mathrm{s}$. Right: corresponding $3 \mathrm{D}$ view of the velocity field on the interior side of the droplet free surface.

equation 6). Note the difference in order of magnitude of the scale bars $\left(\times 10^{-4}, \times 10^{-3}\right.$ and $\left.\times 10^{-2} \mathrm{~m} / \mathrm{s}\right)$. In each case the maximum speed of the flow induced by the air is approximately $1 \%$ of the air speed.

Figure 4 shows the distribution of the solute concentration on the vertical centre plane passing through both droplets, at time $300 \mathrm{~s}$. At the lowest speed, the concentration distribution in the upstream droplet (the rightmost image in Figure 4a) looks very similar to that in droplets under natural convection, since the shear- induced flow is very small. A clear nonuniformity is observable. Generally, it can be noticed that the salt concentration is higher near the upstream sections of the of droplet surfaces, since the highest evaporative flux occurs at these regions. The salt concentration gradually decreases towards the apex region of the droplets surface, and then increases gradually towards the downstream contact-line region, where there is also enhanced evaporation as a result of the sharp vapour concentration gradients there (and all along the contact lines).

Considering the downstream droplet (left-hand droplet in Figure 4a), it is very noticeable that the concentration level in this droplet is significantly less than in the upstream droplet. This is because the water vapour evaporated from the upstream droplet is transported downstream to the other droplet, raising the vapour concentration levels there and hence reducing the local concentration gradients and the associated evaporative flux given by equation (5). When the forced convection speed is increased to $0.2 \mathrm{~m} / \mathrm{s}$, the greater magnitude of the shear-driven circulation pushes the regions of non-uniform concentration closer to the contact lines. The average concentration level is also raised a little by the convection-enhanced evaporation.

At the maximum speed considered, see Figure 4(c), the shear-induced flow within the droplets dominates the gravity-driven flow, and is sufficient to ensure effective mixing of the solute. This results in much more uniform concentration fields in the two droplets (which is still observed when the contour scales are more focused than shown in Figure 4). As expected, the faster air flow increases the evaporation rates of the droplets. However, there is proportionally bigger increase in the evaporation rate of the upstream droplet, and consequently a proportionally bigger difference in the average concentration levels of the two droplets compared to the $0.2 \mathrm{~m} / \mathrm{s}$ case. While the upstream droplet is exposed to 'fresh' air, the downstream droplet sits in the wake of the upstream droplet and consequently experiences both a shielding effect and raised surrounding vapour levels from the downstream water vapour transport.

\subsection{Distribution of water vapour inside the domain}

For a more visual depiction of the 'reach' of the upstream droplet, Figure 5 shows 3D views of the vapour concentration field plotted on the bottom surfaces of the domain and the droplet surfaces. These clearly show how increasing the air speed affects the vapour space between the two droplets, and how the downstream droplet is influenced by the shielding effect of the lead droplet and by the downstream transport from the lead droplet. Consequently, different evaporative behavior is expected between the two droplets. It is worth recalling that periodic conditions are applied in this problem, to represent the transverse array of droplets shown in Figure 1. At zero or low air speed, transverse diffusive transport of water vapour will affect the evaporation rates of the droplets in the domain, particularly at small domain widths. As the droplet wakes show, increasing the air speed will make this transverse diffusive transport within the array negligible. 

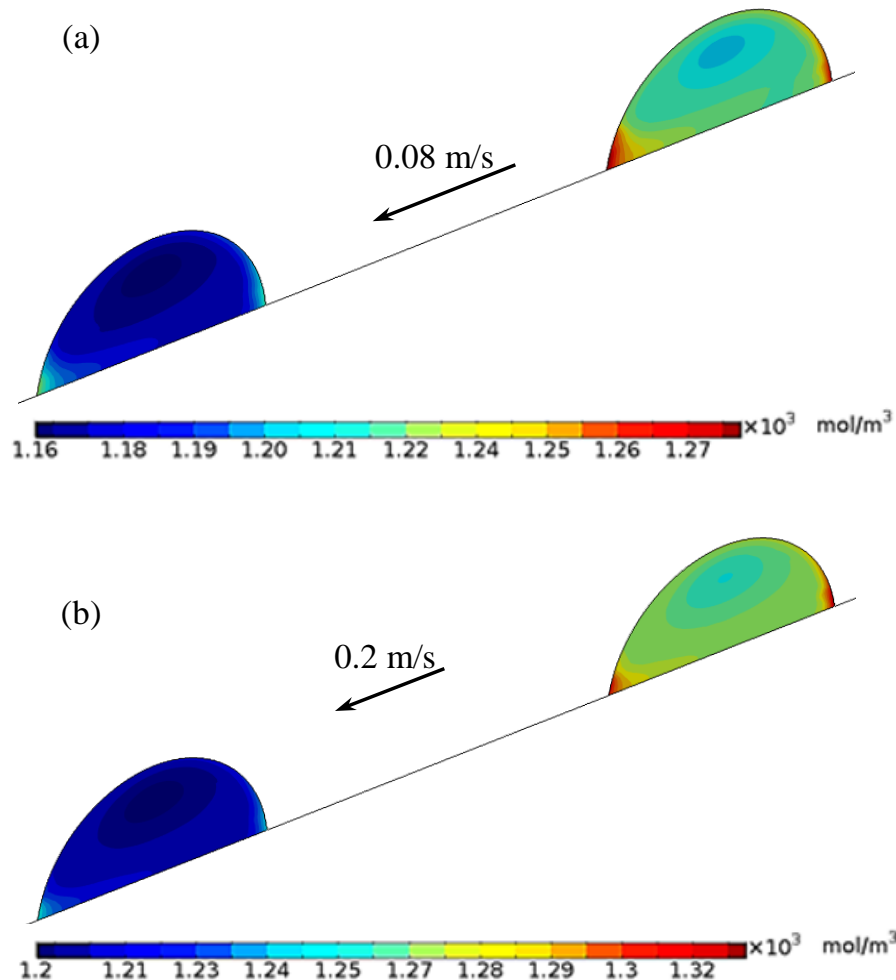

(c)

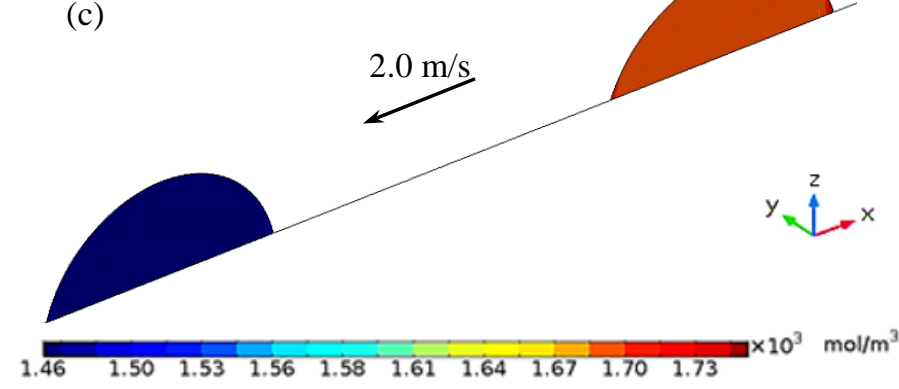

Fig. 4. Contour plot of the solute concentration field (after $300 \mathrm{~s}$ ) at a droplet separation distance of $3 \mathrm{~mm}$ and various air speeds.

\section{Conclusions}

The key observations from this fully-coupled 3D transient finite element study are as follows:

- The forced convective airflow over sessile salt solution droplets produced a circulation flow within the droplets with a maximum speed approximately $1 \%$ of the air speed.

- As air speed increased, the shear-induced circulation becomes sufficient to mix the solution effectively, producing much more uniform concentration distributions in the droplets. Strong variations in concentration still exist near contact lines, but these regions become more localised as air speed increases.

- Downstream vapour transport from the upstream droplet significantly affects the evaporative behaviour of the downstream droplet by raising the

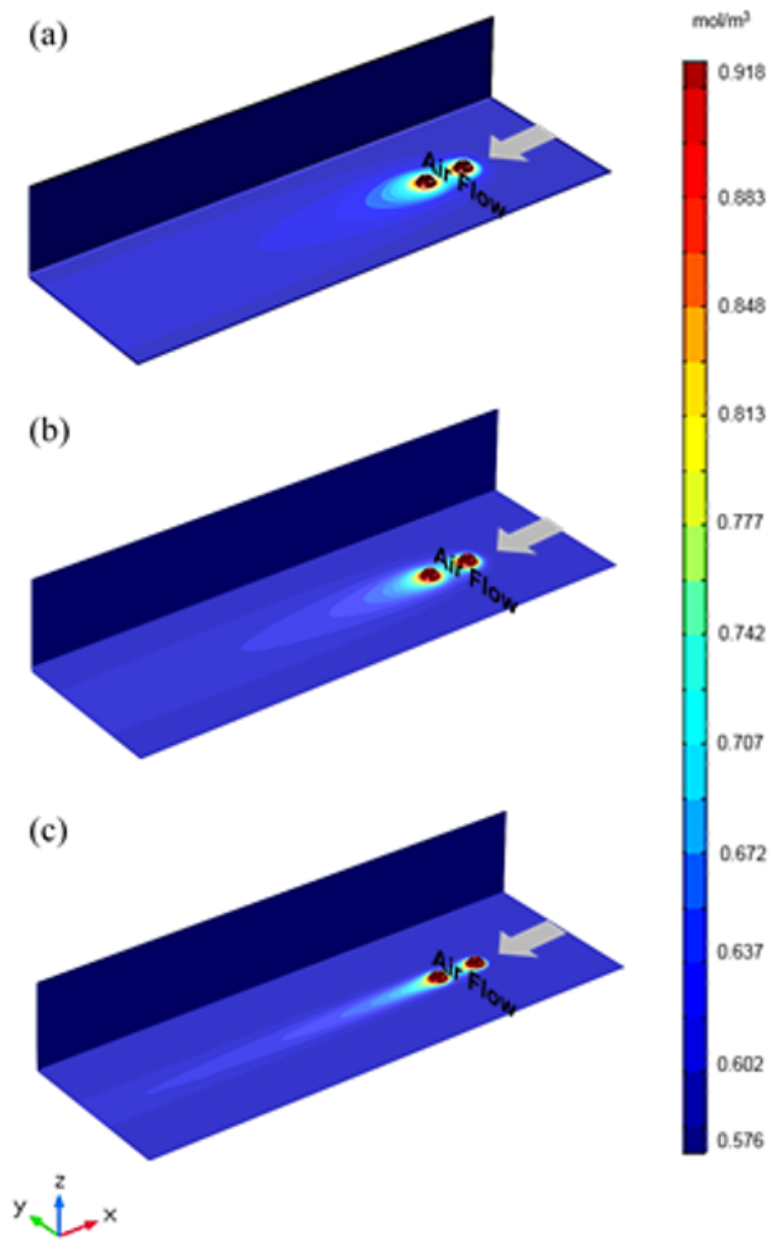

Fig. 5. Vapour concentration on the bottom surfaces of the domain at air speed (a) 0.08, (b) 0.2 , (c) $2 \mathrm{~m} / \mathrm{s}$ and at $3 \mathrm{~mm}$ separations.

surrounding vapour concentration levels and hence reducing concentration gradients.

Omar Al-Rawi thanks the Higher Committee for Education Development in Iraq for funding this work.

\section{References}

1. Kimura, M., et al., Long-Range Ordering of Diblock Copolymers Induced by Droplet Pinning, Langmuir, 19(23), pp. 9910-9913 (2003)

2. Park, J. and J. Moon, Control of Colloidal Particle Deposit Patterns within Picoliter Droplets Ejected by Ink-Jet Printing, Langmuir, 22(8), pp. 35063513(2006)

3. Vehring, R., Pharmaceutical Particle Engineering via Spray Drying, Pharmaceut. Res., 25(5), pp. 999-1022 (2008)

4. Fischer, B.J., Particle Convection in an Evaporating Colloidal Droplet, Langmuir, 18(1), pp. 60-67 (2002)

5. Deegan, R.D., et al., Capillary flow as the cause of ring stains from dried liquid drops, Nature, 389(6653), pp. 827-829 (1997) 
6. Hu, H. and R.G. Larson, Marangoni Effect Reverses Coffee-Ring Depositions, J. Phys. Chem. B, 110(14), pp. $7090-7094$ (2006)

7. Kang, K.H., et al., Evaporation-induced saline Rayleigh convection inside a colloidal droplet, Phys. Fluids, 25(4), pp. 042001 (2013)

8. Savino, R. and R. Monti, Buoyancy and surfacetension-driven convection in hanging-drop protein crystallizer, J. Cryst. Growth, 165(3), pp. 308-318 (1996)

9. Lee, S.J., J. Hong, and Y.-S. Choi, EvaporationInduced Flows inside a Confined Droplet of Diluted Saline Solution, Langmuir, 30(26), pp. 7710-7715 (2014)

10. Pradhan, T.K. and P.K. Panigrahi, Influence of an adjacent droplet on fluid convection inside an evaporating droplet of binary mixture, Colloid. Surface. A, 500, pp. 154-165 (2016)

11. Pradhan, T.K. and P.K. Panigrahi, Evaporation induced natural convection inside a droplet of aqueous solution placed on a superhydrophobic surface, Colloid. Surface. A, 530, pp. 1-12 (2017)

12. $\mathrm{Xu}, \mathrm{G}$, et al., Effect of Salt Concentration on the Motion of Particles near the Substrate in Drying Sessile Colloidal Droplets, Langmuir, 33(3), pp. 685-695 (2017)

13. Pradhan, T.K. and P.K. Panigrahi, Deposition pattern of interacting droplets, Colloid. Surface. A, 482, pp. 562-567 (2015)

14. Davoust, L. and J. Theisen, Evaporation Rate of Drop Arrays within a Digital Microsystem, Procedia Engineering, 47, pp. 1-4 (2012)

15. Davoust, L. and J. Theisen, Evaporation rate of drop arrays within a digital microfluidic system, Sensor. Actuat. B-Chem., 189, pp. 157-164 (2013)

16. Kelly-Zion, P.L., et al., Evaporation of sessile drops under combined diffusion and natural convection, Colloid. Surface. A, 381(1-3), pp. 31-36 (2011)

17. Ait Saada, M., S. Chikh, and L. Tadrist, Numerical investigation of heat and mass transfer of an evaporating sessile drop on a horizontal surface, Phys. Fluids, 22(11): Art. No. 112115 (2010)

18. Misyura, S.Y., Free convection and vapor diffusion of droplet aqueous solutions, Chem. Eng. Res. Des., 126(Supplement C), pp. 153-160 (2017)

19. Misyura, S.Y., Evaporation of a sessile water drop and a drop of aqueous salt solution, Sci. Rep., 7(1), pp. 14759 (2017)

20. Raghuram, S., et al., Numerical study of Marangoni convection during transient evaporation of twocomponent droplet under forced convective environment, Int. J. Heat Mass Tran., 55(25-26), pp. 7949-7957 (2012)

21. Doursat, C., et al., Droplet evaporation on a solid surface exposed to forced convection: Experiments, simulation and dimensional analysis, Int. J. Heat Mass Tran., 113, pp. 1234-1245 (2017)

22. Chakraborty, S., M.A. Rosen, and B.D. MacDonald, Analysis and feasibility of an evaporative cooling system with diffusion-based sessile droplet evaporation for cooling microprocessors, Appl. Therm. Eng., 125, pp. 104 110 (2017)

23. Fan, J., M.C.T. Wilson, and N. Kapur, Displacement of liquid droplets on a surface by a shearing air flow, J. Colloid Interf. Sci., 356(1), pp. 286-292 (2011) 